\title{
NOUVELLE
}

\section{Le foie foetal : source incontournable de cellules souches hépatiques pour la thérapie cellulaire des maladies hépatiques?}

Anne Weber-Benarous, Jean-Étienne Allain

des cellules ovales n'est pas nécessaire. Dans un modèle de tyrosinémie, créé chez la souris par l'invalidation du gène codant pour la fumaryl-acéto-acétate hydrolase (souris $\mathrm{FAH}^{-/-}$), qui provoque l'accumulation de métabolites toxiques pour les hépatocytes, on a pu montrer grâce à des transplantations successives d'hépatocytes normaux, que la capacité de prolifération des hépatocytes excédait 60 divisions [4]. Ainsi, selon la nature du stress subi par le foie, il y aurait

Inserm EMI 00-20,

Hôpital Antoine Béclère,

157, rue de la Porte de Trivaux, 92141 Clamart, France. actuellement le seul traitement de bon
nombre de maladies hépatiques, incluant les maladies métaboliques et les insuffisances hépatocellulaires. La greffe de foie est cependant grevée d'une morbidité et d'une mortalité non négligeables liées au geste chirurgical lui-même, ou aux complications vasculaires et biliaires parfois irréversibles qui lui succèdent. Parallèlement, il existe un décalage croissant entre l'offre de greffons humains et la demande. Ainsi en France, environ $10 \%$ des malades inscrits sur une liste d'attente en vue d'une transplantation hépatique décèdent avant de pouvoir être greffés.

La pénurie de donneurs entraînera obligatoirement une pénurie d'hépatocytes adultes disponibles pour la thérapie cellulaire, d'autant plus que ces cellules ne font guère qu'un ou deux cycles de division en culture et qu'elles sont difficiles à congeler.

La transplantation d'hépatocytes a déjà été réalisée chez quelques enfants atteints de maladies métaboliques; un bénéfice clinique limité et transitoire a été obtenu chez une fillette atteinte d'une maladie de Crigler-Najjar, ce qui prouve que l'approche est réalisable. Ces essais soulèvent aussi un certain nombre de problèmes dus aux propriétés intrinsèques des cellules adultes matures (taille, formation d'agrégats, absence de prolifération). D'autres sources de cellules doivent donc être envisagées. II pourrait s'agir d'hépatocytes fœtaux, mais également de cellules souches issues de tissus adultes ou du foie fœtal.
L'existence de cellules souches dans le foie adulte a été postulée dès 1951. Après des années de débats, les cellules « ovales », petites cellules avec un gros noyau et un cytoplasme réduit, présentes dans le foie des rongeurs, sont maintenant considérées chez ces animaux comme les cellules souches hépatiques adultes. Cette souspopulation hétérogène de cellules épithéliales biliaires, probablement localisées au niveau des canaux de Hering, qui correspondent aux ramifications les plus terminales des canaux biliaires, constitue un compartiment-réserve. Ces cellules sont bipotentes et expriment un certain nombre de marqueurs caractéristiques des cellules hépatocytaires et des cellules biliaires; elles expriment aussi des récepteurs de facteurs de croissance tels c-kit (récepteur du stem cell factor) et c-met (récepteur de I'HGF, hepatocyte growth factor) [1].

Ces cellules, indétectables dans le foie normal, sont recrutées dans des situations où les hépatocytes normaux sont détruits et elles prolifèrent alors pour restaurer la masse hépatique : c'est ainsi qu'elles ont été mises en évidence dans le foie de rongeurs soumis à des carcinogènes ou des agents toxiques (comme la galactosamine), et dans celui de souris transgéniques pour l'antigène T de SV40 $[2,3]$. Mais lorsque la régénération hépatique est induite après une hépatectomie partielle, les hépatocytes résidants prolifèrent dans les lobes restants (hypertrophie compensatrice), et la contribution deux niveaux de réponses de type « cellule souche », l'hépatocyte étant capable de s'auto-renouveler et de rester différencié. Il semblerait que ce concept puisse être étendu à l'homme, puisque la présence de cellules ressemblant aux cellules ovales a été mise en évidence par immuno-histochimie et ultrastructure dans le foie de patients atteints de diverses maladies hépatiques dont l'hépatite chronique [5]. La nature de ces cellules reste à confirmer et elles devront être isolées, purifiées - ce que tentent à l'heure actuelle certaines équipes à partir de tissus hépatiques provenant de patients atteints d'hépatite caractérisées in vitro et amplifiées. Enfin, leur devenir après transplantation dans des modèles animaux devra confirmer leurs potentialités avant d'envisager une utilisation thérapeutique.

Des résultats récents ont montré qu'une sous-population de cellules issues de la moelle osseuse, isolées dans la même fraction phénotypique que les cellules souches hématopoïétiques, et transplantées dans le sinus rétro-orbitaire de souris létalement irradiées, pouvaient donner naissance à des hépatocytes, ce qui suggère que la moelle osseuse pourrait être une troisième source de cellules souches hépatiques [6]. Cependant, il faut noter qu'une telle reconstitution hépatique a été mise en évidence dans une situation expérimentale totalement artificielle, après une transplantation « non convention- 
nelle », que la population de cellules souches n'était pas clonale et qu'il pourrait s'agir d'une sous-population localisée dans la moelle osseuse, mais dont le destin hépatique serait déjà «prédéterminé ». De fait, des résultats très récents montrent que la différenciation de cellules de la moelle osseuse en hépatocytes matures est très inefficace dans des conditions physiologiques [7].

L'utilisation de cellules souches isolées à partir d'organes fœtaux représente une alternative thérapeutique plus immédiate à celle de cellules souches adultes comme l'a récemment démontré l'étude de la greffe de neurones fœtaux chez des patients atteints de maladies neurodégénératives [8]. Cette approche semble particulièrement prometteuse pour le foie. En effet, les cellules fœtales ont des avantages sur les hépatocytes adultes dans une optique de transplantation. Elles sont environ deux fois plus petites (de l'ordre de $15 \mu \mathrm{m}$ par rapport à $30-35 \mu \mathrm{m})$. De ce fait, l'injection des cellules via la veine porte devrait être mieux tolérée et leur migration à travers les sinusoïdes et dans le parenchyme facilitée. En effet, les hépatocytes adultes, y compris chez le primate, restent localisés aux environs des espaces périportaux [9]. Étant donné leur capacité de proliférer, un effet thérapeutique identique à celui obtenu avec des hépatocytes adultes pourrait être obtenu avec un nombre moindre de cellules.

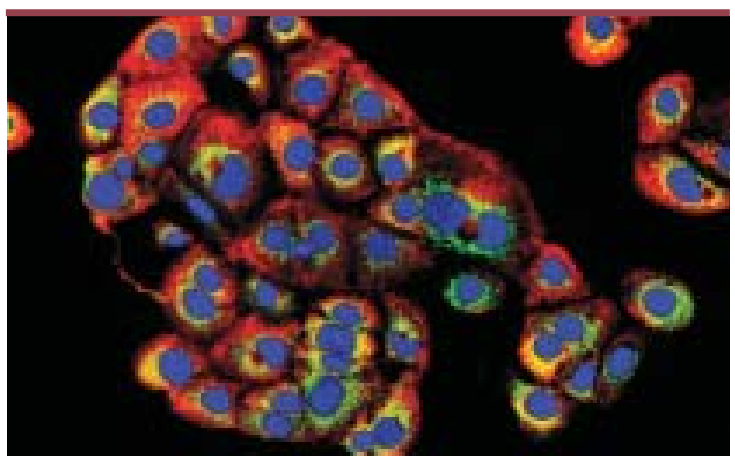

Figure 1. Phénotype bipotent des cellules souches hépatiques fœtales de primate immortalisées. Chaque cellule prolifère et donne naissance à un clone de cellules qui co-expriment l'albumine (vert) et la CK19 (rouge). Les noyaux sont colorés au Dapi (bleu).
Des études réalisées à partir de foies fœtaux de rats ont montré que les hépatoblastes, cellules souches bipotentes, expriment des protéines spécifiques d'hépatocytes (albumine, $\alpha$-fœtoprotéine, HepPar 1) et de cellules biliaires (cytokératines 19 et 7 ). Après transplantation chez le rat, les hépatoblastes sont capables de se différencier non seulement en hépatocytes mais aussi en cellules biliaires lorsqu'ils sont localisés à proximité des canaux biliaires [10].

Notre laboratoire, dont l'objectif est de développer des modèles pré-cliniques de thérapie génique ex vivo et cellulaire de maladies hépatiques chez le primate adulte et fœtal, a défini les conditions adéquates d'isolement, de cryopréservation et de transduction des cellules hépatiques fœtales de macaques. Ce modèle de macaque est en effet particulièrement approprié, car l'anatomie du foie, comme celle du système utéro-placentaire, est très similaire à celle de l'homme. Il en est de même pour la vascularisation hépatique, et une telle similitude n'existe que chez ce mammifère, à la différence des autres gros mammifères - porc, mouton, chien - ce qui est fondamental dans une perpsective de transplantation.

Des banques de cellules hépatiques fœtales de macaque, cryo-préservées, sont réalisées à partir de fœtus de macaques à la fin du $2^{\mathrm{e}}$ tiers de la gestation (90-100 jours), stade auquel la taille du foie et la proportion de cellules hépatiques (par rapport aux cellules hématopoïétiques) permettent d'obtenir un rendement de plusieurs dizaines de millions de cellules.

Dans ces conditions expérimentales, les cellules souches ou hépatoblastes ne représentent qu'un faible pourcentage de la population d'hépatocytes fœtaux.

C'est ainsi qu'in vitro, nous avons caractérisé, au sein des hépatocytes fœtaux, à l'aide de marqueurs spécifiques, une sous-population de petites cellules, particulières par leur cinétique de prolifération, plus rapide que celle des hépatocytes. Afin de pouvoir isoler et amplifier une population clonale d'hépatoblastes, nous avons utilisé un vecteur rétroviral exprimant l'antigène T de SV40 flanqué de sites lox $P$ [11]. Un clone immortel a été isolé après un temps de latence de 9 mois, ce qui représente une fréquence faible, de l'ordre de $5 \times 10^{-8}$, et illustre la différence entre cellules murines et cellules de primates. La lignée dérivée, IPFLS (immortalized primate fetal liver stem cells), n'a pas les caractéristiques d'un phénotype transformé in vitro, et n'exprime pas de potentiel tumoral in vivo après transplantation chez la souris athymique. Cette lignée exprime une très forte activité télomérase, contrairement aux cellules primaires d'origine, dans lesquelles l'activité télomérase disparaît après 3 jours de culture. La capacité de prolifération de ces cellules est a priori illimitée puisque nous maintenons la lignée en culture depuis 3 ans. Le phénotypique bipotent de la lignée semble stable puisque les cellules expriment à la fois des marqueurs hépatocytaires (albumine, $\alpha$-fœtoprotéine, cytokératines $8 / 18$ ) et de cellules biliaires (CK19) (Figure 1). Enfin, leur caractère immortalisé est bien dû à l'expression de l'antigène $T$ puisque son excision, par l'intermédiaire de la recombinase CRE transférée à l'aide d'un vecteur viral, entraîne l'arrêt de croissance des cellules.

La fonctionnalité de ces cellules a été testée après leur transplantation, via la veine porte, dans le foie de souris athymiques ayant subi l'ablation de $50 \%$ de la masse hépatique. La quantité de cellules injectées représente environ $1 \%$ de la masse hépatocytaire de l'animal. Au bout de 8 jours, ces cellules sont retrouvées essentiellement autour des espaces portes, et environ $50 \%$ sont intégrées dans le parenchyme, ce qui corrobore les données obtenues avec les hépatocytes normaux. Les cellules IPFLS n'expriment plus la CK 19, témoin d'un phénotype biliaire mais elles 
expriment toujours l'albumine (Figure 1). De plus, un certain nombre d'entre elles ont perdu leur caractère mononucléé, caractéristique des cellules fœtales, et acquis celui de cellule binucléée, caractéristique des hépatocytes adultes. Ces résultats laissent penser que les cellules souches fœtales hépatiques transplantées dans un foie adulte répriment rapidement les fonctions fœtales.

Trois semaines après transplantation, il s'avère que l'avantage prolifératif de ces cellules observé in vitro ne leur confère pas un meilleur avantage intégratif que celui des hépatocytes normaux in vivo. Ces résultats sont intéressants en ce qu'ils suggèrent un rôle important du microenvironnement dans un foie quiescent. Cependant, il faut noter que le devenir des cellules transplantées ne peut être suivi à moyen terme dans les souris athymiques. L'utilisation de souris immunodéficientes $\mathrm{rag}^{-/-} \times \gamma_{c^{-/-}}$développées par J. DiSanto [12] permettra d'analyser le devenir de ces cellules à long terme et d'évaluer leur différenciation en fonction de leur distribution dans le parenchyme, en particulier si les cellules intégrées au voisinage des canaux biliaires continuent d'exprimer des marqueurs biliaires au détriment des fonctions hépatocytaires. II sera aussi intéressant d'évaluer si l'avantage prolifératif des cellules transplantées persiste et quelle sera l'étendue de la contribution de ces cellules à la repopulation hépatique après l'induction d'un signal de régénération. Par ailleurs cette lignée de primate constitue un outil intéressant pour des approches pré-cliniques de transplantation dans notre modèle de macaque : l'objectif sera de définir les conditions de transplantation et de prise de greffe non seulement en post-natal mais également in utero et d'évaluer le chimérisme du foie receveur.

\section{Perspectives de thérapie cellulaire pour certaines maladies hépatiques}

La mise au point d'un tel modèle a jeté les bases d' études réalisées à partir de cellules hépatiques fœtales humaines prélevées à un stade précoce. En effet, l'isolement chez l'Homme, d'une telle population de cellules souches bipotentes, capables de se différencier en hépatocytes et en cellules biliaires, et susceptibles de proliférer permettrait d'évaluer réellement leurs propriétés et

leur fonctionnalité in vivo chez l'animal. La facilité avec laquelle les cellules fœtales peuvent être congelées par opposition aux hépatocytes adultes permet d'envisager la constitution de banques, selon des procédés bien définis, ce qui facilitera le criblage de ces cellules en termes de sécurité biologique mais aussi leur typage tissulaire.

L'analyse de leur différenciation in vivo sera l'élément déterminant dans la perspective d'une utilisation des cellules souches fœtales en thérapie cellulaire, que ce soit par allo-transplantation ou transplantation autologue de cellules génétiquement modifiées.

L'utilisation de cellules humaines immortalisées selon un système conditionnel pourrait également constituer une approche thérapeutique. En effet, de telles cellules pourront être amplifiées à grande échelle et leur différenciation induite in vitro. Elles pourraient ensuite être encapsulées et implantées dans la cavité péritonéale ou servir à la mise au point de nouveaux foies bioartificiels. $\diamond$ Fetal liver: an obligatory source of stem cells for a therapeutic approach of liver disease?

\section{REMERCIEMENTS}

\section{RéFÉRENCES}

1. Alison M. Liver stem cells: a two compartment system. Curr Opin Cell Biol 1998; 10 : 710-5.

2. Lazaro CA, Rhim JA, Yamada $y$, Fausto N. Generation of hepatocytes from oval cell precursors in culture. Cancer Res 1998 ; 58 : 5514-22.

3. Bennoun M, Rissel M, Engelhardt N, Guillouzo A, Briand $P$, Weber-Benarous $A$. Oval cell proliferation in early stages of hepatocarcinogenesis in simian virus 40 large $T$ transgenic mice. Am J Pathol 1993 ; 143 : 1326-36.

4. Overturf $K$, al-Dhalimy $M$, Ou CN, Finegold M, Grompe M. Serial transplantation reveals the stem-cell-like regenerative potential of adult mouse hepatocytes. Am J Pathol 1997 ; 151 :

1273-80.

5. Libbrecht L, Desmet V, Van Damme B, Roskams T.

Deep intralobular

extension of human hepatic progenitor cells

correlates with parenchyma inflammation in chronic viral hepatitis: can progenitor cells migrate? Pathol 2000 ; 192 : 373-8.

6. Lagasse $\varepsilon$, Connors H, AlDhalimy $M$, et al. Purified hematopoietic stem cells can differentiate into hepatocytes in vivo. Nat Med 2000 ; 6 : 1229-34.

7. Mallet VO, Mitchell C, Mezey $\varepsilon$, et al. Bone marrow transplantation in mice leads to a minor population of hepatocytes that can be selectively amplified in vivo. Hepatology 2002 ; 35 : 799-04.

8. Peschanski M. 10 years of substitution therapy for neurodegenerative diseases using fetal neuron grafts: a positive outcome but with questions for the future. $J$ Soc Biol 2001 ; 195 : 51-5.

9. Andreoletti M, Loux N, Vons $C$, et al. Engraftment of autologous retrovirally transduced hepatocytes after intraportal transplantation into nonhuman primates: implication for ex vivo gene therapy. Hum Gene Ther 2001 ; 12 : 169-79.

10. Kubota H, Reid LM. Clonogenic hepatoblasts, common precursors for hepatocytic and biliary lineages, are lacking classical major
Ce travail a été réalisé avec le soutien de l'AFM et de la FRM.

histocompatibility complex class I antigen. Proc Natl Acad Sci USA 2000 ; 97 : 12132-7.

11. Allain JE, Dagher I, Mahieu-Caputo D, et al. Immortalization of a primate bipotent epithelial liver stem cell. Proc Natl Acad Sci USA 2002 ; 99 : 3639-44.

12. Goldman JP, Blundell MP, Lopes L, Kinnon C, Di Santo JP, Thrasher AJ. Enhanced human cell engraftment in mice deficient in RAG2 and the common cytokine receptor gamma chain. BrJ Haematol 1998 ; 103 : 335-42. 